\title{
Avaliação das ações de detecção de casos de tuberculose na atenção primária
}

\author{
Evaluation of detection actions for tuberculosis cases in primary care
}

Roxana Isabel Cardozo-Gonzales ${ }^{1}$, Pedro Fredemir Palha ${ }^{2}$, Jenifer Harter ${ }^{3}$, Edith Alarcon ${ }^{4}$, Lílian Moura de Lima ${ }^{5}$, Jessica Oliveira Tomberg ${ }^{6}$

\footnotetext{
${ }^{1}$ Enfermeira, Doutora em Enfermagem em Saúde Pública. Professor Adjunto da Faculdade de Enfermagem e Obstetrícia da Universidade Federal de Pelotas (UFPel). Pelotas, RS, Brasil. E-mail: rcardozogonzales@yahoo.com.

${ }^{2}$ Enfermeiro, Doutor em Enfermagem em Saúde Pública. Professor Associado da Escola de Enfermagem de Ribeirão Preto da Universidade de São Paulo. Ribeirão Preto, RS, Brasil. E-mail: ppalha@eerp.usp.br.

${ }^{3}$ Enfermeira, Mestre em Enfermagem. Discente do Programa de Pós-Graduação em Enfermagem, nível Doutorado, da UFPel. Professor Assistente da Universidade Federal do Pampa. Pelotas, RS, Brasil. E-mail: jeniferharter@hotmail.com.

${ }^{4}$ Enfermeira, Mestre em Saúde Pública. Consultora do Departamento de TB-HIV América Latina Região. Lima, Peru. E-mail: ealarcon@theunion.org.

${ }^{5}$ Enfermeira, Mestre em Enfermagem. Discente do Programa de Pós-Graduação em Enfermagem, nível Doutorado, da UFPel. Professor do Instituto Federal do Paraná. Pelotas, RS, Brasil. E-mail: lima.lilian@gmail.com.

${ }^{6}$ Enfermeira, Mestre em Enfermagem. Discente do Programa de Pós-Graduação em Enfermagem, nível Doutorado, da UFPel. Pelotas, RS, Brasil. E-mail: jessicatomberg@hotmail.com.
}

\section{RESUMO}

Objetivou-se avaliar as ações de detecção de casos de tuberculose nos serviços de atenção primária à saúde de um município do Sul do Brasil. Estudo exploratório, quantitativo, com 76 enfermeiros. Utilizou-se a análise de correspondência múltipla para identificar as associações entre ações para deteç̧ão de casos (busca de sintomáticos respiratórios, educação em saúde, discussões sobre tuberculose com lideranças comunitárias) e as características dos serviços (com ou sem estratégia de saúde da família), usando o software Statistica 12. As unidades com estratégia de saúde da família apresentaram melhor resultado para deteç̧ão de casos ao associarem-se com a realização regular das ações, enquanto as unidades em que não há equipes de saúde da família a realização da detecção associou-se à infrequência das ações. Conclui-se que existem diferenças entre ambos os serviços em relação ao desempenho das ações de detecção de casos.

Descritores: Tuberculose; Avaliação de Serviços de Saúde; Saúde Pública.

\section{ABSTRACT}

The objective was to assess the detection actions of tuberculosis cases in primary care in a city in the South of Brazil. An exploratory, quantitative study, with 76 nurses. A multiple correspondence analysis was used to identify associations between actions to detect cases (search of respiratory symptoms, health education, discussions about tuberculosis with community leaderships) and the services' characteristics (with or without the family health strategy), using the Statistica 12 software. The units with family health strategy presented better result to detect cases when associated with regular actions, while in units without family health teams the performing detection was associated to infrequency of actions. It was concluded that differences exist in both services regarding performance of case detection actions.

Descriptors: Tuberculosis; Health Services Evaluation; Public Health. 


\section{INTRODUÇÃO}

O controle da tuberculose (TB) ainda permanece como grande desafio para o sistema de saúde brasileiro. O Brasil integra o grupo de 22 países com a mais elevada carga desta doença. Embora o país tenha sido avaliado com alta taxa de detecção de casos no ano de 2012, ainda existem dificuldades para o alcance das metas de controle da doença, visto que a taxa de sucesso de tratamento, neste mesmo ano, foi de apenas de $72 \%^{(1)}$. Salienta-se que as três esferas de governo no país - União, Estados e Municípios detêm responsabilidades no controle da tuberculose.

A detecção de casos, o mais precocemente, e o tratamento oportuno, são estratégias essenciais para o controle da TB. Destaca-se a importância central dos serviços de atenção primária à saúde (APS) no desenvolvimento dessas estratégias incluindo o tratamento diretamente observado e a contrarreferência de unidades secundárias e terciárias ${ }^{(2)}$.

A eficiência na busca de casos e a agilidade no início do tratamento da TB pelos serviços da APS contribuem expressivamente para o uso mais eficiente dos recursos humanos, o menor ônus para o sistema, a prevenção da transmissão ${ }^{(3)}$ e a redução do sofrimento das pessoas e famílias acometidas pela TB.

Reconhece-se a APS como a principal porta de entrada e o centro organizador do sistema de saúde brasileiro. A resolutividade das necessidades em saúde, a coordenação da atenção e a responsabilização pela população adscrita são as três funções essenciais a esta organização do sistema (Portaria GM no 2.488/2011), capaz de produzir impacto no perfil epidemiológico e no controle da TB. Considera-se que o foco da atenção primária em ações de promoção, prevenção e identificação das necessidades de saúde da família na comunidade favorecem operacionalmente a detecção de casos.

Contudo, evidencia-se o descompasso entre a política de controle da TB e as práticas de saúde nos serviços de atenção primária. Visto que, estes serviços, apesar de apresentarem estrutura adequada e disponibilidade de materiais para solicitação de baciloscopia de escarro e realização do diagnóstico da $\mathrm{TB}^{(4)}$, ainda realizam menos diagnósticos em relação às unidades de pronto atendimento e aos serviços especializados. Embora seja adotada como a principal estratégia de detecção de casos, a prática da busca de sintomáticos respiratórios (BSR) nos serviços de saúde e na comunidade é incipiente.

$\mathrm{Na}$ literatura atual identificam-se produções sobre a problemática da detecção de casos de TB, dando ênfase, prioritariamente, as barreiras no acesso aos serviços de saúde $^{(5-6)}$ e a realização dos exames diagnósticos ${ }^{(7-8)}$. Evidenciando a necessidade de estudar os aspectos relacionados à avaliação do desenvolvimento de ações, inerentes ao trabalho da equipe de saúde da atenção primária, no território, para efetivar o controle da TB.

Diante do exposto, realizou-se a presente investigação elegendo como local de estudo um município de grande porte populacional, situado na metade sul do Brasil, considerado pelo Ministério da Saúde como prioritário para as ações de controle da TB. As ações de detecção de casos no município são desenvolvidas por todos os profissionais de saúde das unidades de atenção primária. Enquanto que o tratamento da doença é realizado em uma unidade ambulatorial especializada, constituída por uma equipe de saúde composta por dois médicos pneumologista, uma assistente social e uma recepcionista. Embora exista essa organização para a atenção à doença no município, o impacto no controle ainda é insuficiente, visto que em 2014 foram notificados 272 casos da doença, sendo que desses 199 eram casos novos, representando uma incidência de 58,17 casos por 100.000 habitantes.

Objetivou-se avaliar as ações de detecção de casos de TB nos serviços de atenção primária à saúde de um município do Sul do Brasil, utilizando como fonte de dados os enfermeiros das unidades em estudo, em virtude do envolvimento deste profissional com as ações de cuidado, de assistência e de gerência dos serviços de APS $^{(9)}$ e execução das ações de controle da TB ${ }^{(2)}$. Portanto 
considera-se um informante-chave para avaliar as ações de detecção de casos de TB desenvolvidas pela equipe de saúde.

\section{MÉTODOS}

Estudo exploratório de abordagem quantitativa, realizado nas unidades de APS de um município de grande porte populacional localizado no Sul do Brasil com aproximadamente 342.053 habitantes ${ }^{(10)}$, o qual possui 51 unidades de APS, sendo 34 unidades básicas de saúde tradicionais (UBST) e 17 unidades saúde da família (USF) com 41 equipes de saúde da família implantados atingindo a cobertura de $42,94 \%$ da população ${ }^{(11)}$.

Dos 84 enfermeiros atuantes na APS do município, quatro não foram considerados no estudo devido à licença saúde, maternidade e férias no período de abril a agosto de 2012, quando ocorreu a coleta de dados. Houve três perdas referentes aos profissionais que não foram encontrados na unidade em seus turnos de trabalho por no mínimo quatro tentativas e um se recusou a participar do estudo.

Desta forma, o estudo foi constituído por 76 sujeitos. As entrevistas foram realizadas no local e turno de trabalho dos enfermeiros, de acordo com a disponibilidade deles. Estes profissionais responderam a um questionário estruturado elaborado com base em um instrumento que avalia a atenção à TB no Brasil ${ }^{(12)}$, prétestado com 20 profissionais de saúde de município próximo ao local de estudo.

As variáveis selecionadas correspondem às ações inerentes ao trabalho desses profissionais de saúde nos serviços para identificação dos casos de TB (frequência nas ações de busca de sintomático respiratório no serviço de saúde - BSRS, busca de sintomático respiratório na comunidade - BSRC, educação em saúde sobre TB no serviço - EDS, educação em saúde sobre TB na comunidade - ESC, discussões com lideranças comunitárias sobre TB - RDL). Cada uma dessas variáveis recebeu adicionais numéricos representativos das opções de resposta para a frequência no desenvolvimento das ações ao serem representadas no mapa perceptual na Análise de Correspondência Múltipla. O numero 1 significou infrequência; 2 frequência regular; e 3 frequentemente.

A análise de correspondência múltipla buscou identificar as associações entre as ações de detecção de casos desenvolvidos pela unidade de saúde (variáveis ativas) e as características destes serviços em que os profissionais atuavam (variáveis passivas) sem a necessidade de uma relação causal prévia ${ }^{(13)}$. Foi utilizado o decrescimento dos autovalores para definição das dimensões de análise, considerando, portanto, as dimensões que antecederam descidas significativas nos autovalores, uma vez que apresentam maior variabilidade dos dados. Tais dimensões representam um conjunto de variáveis, deste conjunto excluíram-se as variáveis com menores contribuições absolutas $(\operatorname{Cos} 2<0,20)$ por apresentarem instabilidade no mapa perceptual. A contribuição absoluta é o somatório das contribuições de cada opção de resposta de uma variável à dimensão.

Para análise do Mapa Perceptual da ACM foram inseridas as variáveis passivas "modalidade de serviço de saúde (ESF/sem ESF)" e "frequência com que recebe SR (infrequente/regularmente/frequentemente)". Destacase que estas não têm contribuição na dispersão dos pontos no mapa, porém auxiliam na interpretação dos resultados gráficos ${ }^{(14)}$.

Os dados foram analisados usando Statistica 12 (Statsoft, Tulsa, OK, USA). O estudo obteve aprovação do Comitê de Ética em Pesquisa da Universidade Católica de Pelotas, parecer no 2009/04. Os preceitos éticos foram respeitados e os entrevistados assinaram o Termo de Consentimento Livre e Esclarecido para participar do estudo.

\section{RESULTADOS}

Na Tabela 1 estão apresentadas as variáveis e os respectivos valores dos cossenos para as dimensões 1 e 2 . A dimensão 1 apresentou predominantemente variáveis 
relacionadas às ações de BSR e de educação em saúde e discussões com as lideranças comunitárias sobre TB, e explica $31,65 \%$ da variabilidade dos dados. Enquanto a dimensão 2 apresenta a variável relacionada com o desenvolvimento de atividades de educação em saúde sobre TB na própria unidade explicando $23,73 \%$ da variabilidade dos dados.

Tabela 1: Contribuição absoluta das variáveis à dimensão. Município da região Sul do Brasil, de abril a agosto de 2012.

\begin{tabular}{llll}
\hline Variável & Cos $^{2}$ Dimensão 1 & Cos $^{2}$ Dimensão 2 & Dimensão \\
\hline BSR - Busca de SR no Serviço de Saúde & 0,469 & 0,374 & 1 \\
BSC - Busca de SR na comunidade & 0,592 & 0,240 & 1 \\
EDS - Educação em saúde sobre TB no Serviço & 0,443 & 0,469 & 2 \\
ESC - Educação em saúde sobre TB na Comunidade & 0,579 & 0,471 & 1 \\
RDL - Realiza discussões com lideranças comunitárias sobre TB & 0,520 & 0,348 & 1 \\
\hline
\end{tabular}

No mapa perceptual (Figura 1) estão representadas as variáveis pertencentes a cada dimensão localizadas por meio de suas coordenadas. No quadrante superior esquerdo (Q-IV) encontram-se as variáveis associadas com a infrequente realização de ações de detecção de casos (BSRS, BSRC, EDS, ESC e RDL). Estas avaliações correspondem às unidades de saúde sem ESF e que não recebem sintomáticos respiratórios com frequência.

Figura 1: Mapa Perceptual das ações de detecção de casos de TB na atenção primária de um município do Sul do Brasil.

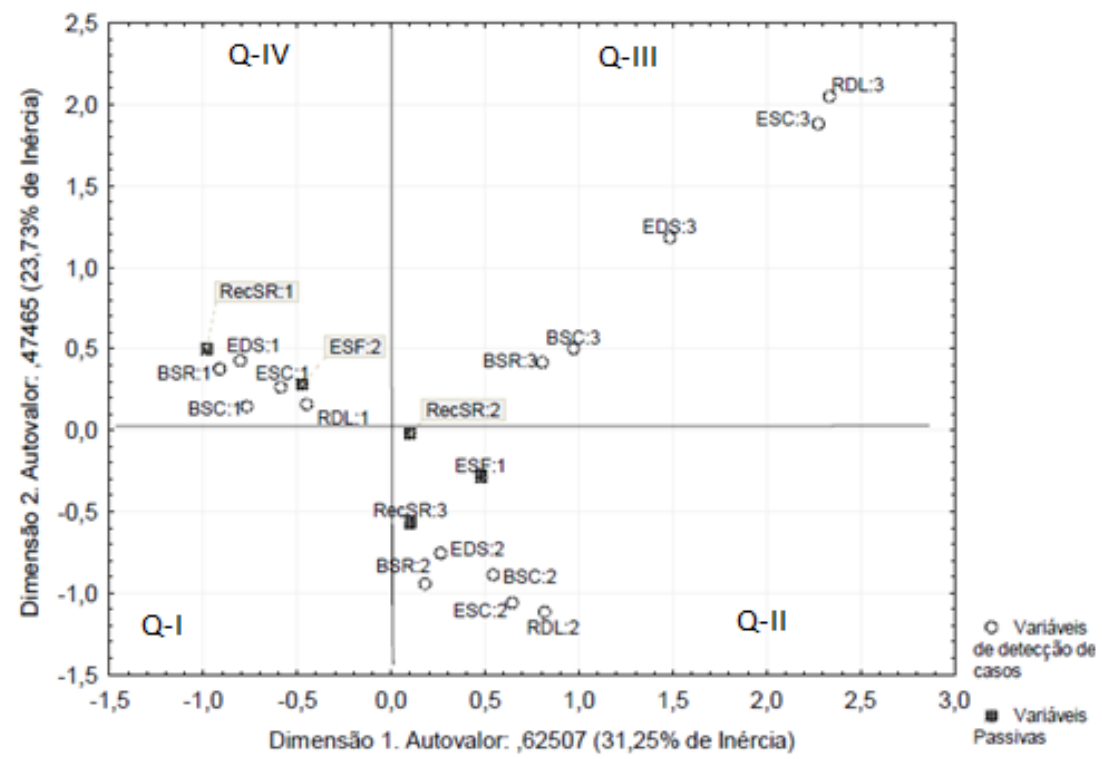

Legenda:

\begin{tabular}{|l|}
\hline ESF1: Unidade de saúde com Estratégia Saúde da Família \\
ESF2: Unidade de saúde sem Estratégia Saúde da Família \\
\hline RecSR1: recebimento infrequente de sintomáticos respiratórios \\
RecSR2: recebimento regular de sintomático respiratório \\
RecSR3: recebimento frequente de sintomáticos respiratórios \\
\hline BSC1: busca infrequente de sintomático na comunidade \\
BSC2: busca regular de sintomático respiratório na comunidade \\
BSC3: busca frequente de sintomático na comunidade \\
\hline BSS1: Busca infrequente de sintomático no serviço de saúde \\
BSS2: busca regular de sintomático no serviço de saúde \\
BSS3: busca frequente de sintomático no serviço de saúde \\
\hline EDS1: educação em saúde no serviço infrequente \\
EDS2: educação em saúde no serviço regularmente \\
EDS3: educação em saúde no serviço frequente \\
\hline ESC1: educação em saúde na comunidade infrequente \\
ESC2: educação em saúde na comunidade regularmente \\
ESC3: educação em saúde na comunidade frequente \\
\hline RDL1: discussão infrequente sobre TB com lideranças comunitárias \\
RDL2: discussão sobre TB com lideranças comunitárias regularmente \\
RDL3: discussão frequente sobre TB com lideranças comunitárias. \\
\hline
\end{tabular}


No quadrante inferior direito (Q-II) se localizam as variáveis associadas com o desenvolvimento regularmente frequente de ações de detecção de casos em unidades caracterizadas como unidades com estratégia de saúde da família e que recebem frequentemente os sintomáticos respiratórios.

As avaliações relacionadas ao desenvolvimento frequente das ações de detecção de casos encontram-se no quadrante superior direito (Q-III). Estas avaliações não estão relacionadas com o recebimento frequente de sintomáticos respiratórios.

\section{DISCUSSÃO}

A detecção de casos é uma atividade complexa e representa um grande desafio para os serviços de saúde, em termos de planejamento do número de sintomáticos a avaliar $^{(15)}$ e de frequência no desenvolvimento das ações $^{(16)}$, as quais ainda vem apresentando-se como deficientes em vários contextos sanitários do país ${ }^{(5)}$. Realidade também identificada neste estudo.

A complexidade dessa ação na atenção à saúde reside na necessidade de uma abordagem profissional qualificada, comprometida com o sujeito e com o controle da TB, pautada no diálogo, na formação de vínculo, capaz de gerar segurança para o sujeito perante sua necessidade de resolver seu problema de saúde, de não se sentir ameaçado por ser portador de uma doença estigmatizante e de alta transmissibilidade. É essencial que se estabeleça uma relação de coparticipação no processo de atenção que possibilite a aceitabilidade do sujeito com sintomas da TB das ações necessárias à descoberta da doença e a correspondência de saberes, desejos e necessidades entre o sujeito que busca atendimento e o profissional de saúde que o recebe.

O Programa Nacional de Controle da TB recomenda que as ações de identificação de sintomáticos respiratórios sejam realizadas permanentemente pelos serviços de saúde e principalmente pelos serviços de APS $^{(2)}$. Desta forma, a infrequência da BSR, de educação em saúde tanto no serviço quanto na comunidade e de discussões com lideranças comunitárias, observadas no estudo, comprometem a identificação ativa (busca na comunidade) e passiva (busca no serviço de saúde) dos casos de TB e a visibilidade da doença como importante problema de saúde na população. Em decorrência disso, serviços primários de saúde recebem poucos sintomáticos respiratórios e a população desconhece a oferta das ações de detecção de casos nestes serviços. Por consequência favorece-se a permanência da doença $^{(16)}$.

As dificuldades que impedem a realização da detecção eficaz de casos de TB estão relacionadas aos aspectos operacionais do serviço de saúde (forma de organização para detectar e tratar os casos) ${ }^{(5)}$ com impacto no tempo entre a identificação do sintomático respiratório e o diagnóstico ${ }^{(17)}$. Ainda a modalidade de atenção às pessoas (unidades tradicionais e unidades com ESF) e a qualificação dos profissionais podem influenciar no desempenho desta ação nos serviços da $\operatorname{APS}^{(5)}$.

A infrequência nas ações de detecção de casos nas unidades caracterizadas como tradicionais, neste estudo, pode ser atribuída à organização para a atenção às pessoas neste tipo de serviço. Essas unidades, em geral, possuem um território amplo, recebendo acentuada e variada demanda de usuários, implicando em atuarem predominantemente dentro do serviço de saúde em detrimento das ações externas à unidade. Assim, as ações no território, de BSR e de caráter coletivo, como a educação em saúde e reuniões com lideranças comunitárias, ficam aquém do necessário para promover a identificação precoce dos casos da doença presentes nessas unidades.

Ainda com relação ao desenvolvimento incipiente de ações voltadas para a TB nas unidades de saúde estudadas, destaca-se que, algumas características da organização dos serviços de saúde para a oferta da atenção se constituem em barreiras para a utilização de serviços de saúde. Dentre estas, citam-se os horários restritos de atendimento, a falta de acolhimento, as filas e os longos tempos de espera por atendimento presentes 
no contexto sanitário em estudo e já descritos na literatura $^{(18)}$. Tais dificuldades impostas no acesso a APS resultam em perda de oportunidades para identificar pessoas com sintomas da doença, portanto repercutem no tempo de diagnóstico, de início do tratamento, no agravamento clínico e contágio da doença.

A BSR realizada pela equipe de saúde tanto no serviço quanto na comunidade são estratégias essenciais para identificação dos casos. Perguntar ativamente sobre a presença de tosse para todos os adultos que comparecem à unidade de saúde pode aumentar significativamente o número de casos diagnosticados de $\mathrm{TB}^{(19)}$. Estima-se que 5\% das pessoas maiores de 15 anos que buscam o serviço de saúde para primeira consulta podem apresentar tosse por mais de três semanas, devendo nestes casos ser investigada a hipótese de $\mathrm{TB}^{(2)}$ essa estimativa mostra a necessidade imperativa de execução desta ação.

Tal estratégia quando realizada também na comunidade tende a ampliar a identificação precoce dos sintomáticos, pois ao considerar que os usuários procuram os serviços de saúde em consequência do agravamento dos sintomas da TB, aguardar a busca passiva pelo serviço de saúde provocaria o retardo no diagnóstico ${ }^{(20)}$.

A diferença no desenvolvimento da BSRC entre as unidades, infrequente nos serviços sem ESF e regularmente realizados por aqueles que possuem esta estratégia, pode ser decorrente da presença de agentes comunitários de saúde nas unidades de saúde da família, já que esta ação é realizada por esse profissional nas visitas aos domicílios ${ }^{(21)}$. Desta forma, o investimento na sustentabilidade deste trabalhador, em termos quantitativos, e de qualificação, na abordagem à TB, desvela-se como uma iniciativa para impactar no desenvolvimento da detecção de casos. Assim como, é elementar o investimento na ampliação do Programa de Agentes Comunitários de Saúde (PACS) para todas as unidades de APS, fortalecendo assim a estratégia de busca ativa de casos e o consequente efetivo controle da doença.
Em relação à realização de educação em saúde sobre TB, estudo realizado com enfermeiros da Paraíba, ressalta as limitações do espaço físico das unidades de saúde como barreira na execução desta ação ${ }^{(20)}$. Entretanto, as equipes de saúde podem usufruir de espaços comunitários para realizar as ações educativas, tais como igrejas, escolas e associações de bairro. Esta estratégia tende a potencializar o efeito da ação educativa, pois coloca os profissionais de saúde no espaço comunitário, aproximando-os da realidade social destes indivíduos, e potencializando o fortalecimento do vínculo. Destaca-se que a infrequência na realização de ações educativas, tanto a nível interno da unidade, mas principalmente no espaço comunitário, prejudica a capacidade de autoatenção do sujeito e da comunidade, devido à ausência de informações sobre ações disponíveis para o diagnóstico da TB e as formas de obtê-las nos serviços de saúde. Em consequência, a procura pelos serviços ocorre após longo período do aparecimento dos sintomas, agravando o quadro clínico da doença e elevando a possibilidade de transmitir a TB no ambiente social e de trabalho, o que contribui com a manutenção do número de casos novos.

A similaridade na distribuição dos usuários na procura por outros serviços de saúde além da APS e o tempo superior a 30 dias para obter o diagnóstico da doença quando utilizado serviços da APS, observados em estudo multicêntrico ${ }^{(16)}$, podem representar resultados decorrentes da infrequência e do desenvolvimento regular das ações de detecção de casos encontrados neste estudo.

O desempenho incipiente das ações de detecção de casos observado no estudo coaduna aos demais problemas vivenciados na APS brasileira na atualidade. Problemas de gestão com repercussão na estrutura física, na suficiência e perfil de trabalhadores, no acesso oportuno aos recursos e na efetividade das ações de saúde ${ }^{(18)}$ influenciam no desempenho desses serviços no enfrentamento e controle dos problemas de saúde da população, sendo um deles a TB. 
Em relação à discussão com os lideres comunitários cabe destacar a importância dessa ação, uma vez que incentiva a inclusão e envolvimento da sociedade civil na luta contra a TB. A participação social na gestão da saúde foi gestada nos diferentes níveis de governo por meio do aparato jurídico-legal (Lei 8.080/90, no Capítulo II e Art. 7ㅇ). Entretanto, no que diz respeito à TB, esta participação se apresenta como insuficiente e deficiente em alguns sistemas locais de saúde. Esta realidade pode ocorrer em decorrência da invisibilidade da doença como grave problema de saúde pelos gestores e profissionais de saúde ou pelo despreparo no enfrentamento das doenças com coparticipação comunitária.

Investimentos em iniciativas de ampliação das ações de controle da doença, especificamente as de detecção de casos de TB, são indispensáveis no contexto estudado frente ao desenvolvimento infrequente e regular das ações de BSR, educação em saúde sobre TB e promoção da participação da comunidade no debate sobre a temática.

\section{CONCLUSÃO}

De modo geral os resultados identificados no estudo mostram diferenças entre ambos os serviços em relação ao desempenho das ações de detecção de casos. Ressalta-se que todas as variáveis utilizadas no estudo representam recomendações vigentes para o controle da TB, portanto deveriam ser atendidas por todas as unidades de APS. Diante disso, investir na qualificação dos recursos humanos é urgente e deve ser uma ação contínua, para melhores condições de trabalho e alteridade de cada membro da equipe, do gerente e do cidadão, permitindo a suspeita da doença ser possível em qualquer serviço de saúde ou ambiente comunitário.

\section{REFERÊNCIAS}

1. WHO. Global tuberculosis report 2014. 2014.

2. Ministério da Saúde (Brasil). Secretaria de Vigilância em Saúde. Departamento de Vigilância Epidemiológica. Manual de recomendações para o controle da TB no Brasil. Brasília: Ministério da Saúde, 2011.

3. Williams $G$, Alarcon $E$, Jittimanee $S$, Walusimbi $M$, Sebek $M$, Berga $E$, Villa TS. Identifying an active case of TB. Int J Tuberc Lung Dis. 2008;12(4):381-385.

4. Andrade RLP, Scatolin BE, Wysocki AD, Beraldo AA, Monroe AA, Scatena LM et al. Tuberculosis diagnosis: primary health care or emergency medical services?. Rev. Saúde Pública [periódico na Internet]. 2013;47(6):11491158.

5. Brunello ME , Andrade RL, Monroe AA , Arakawa T, Magnabosco GT, Orfão NH, et al. Tuberculosis diagnostic pathway in a municipality insouth-eastern Brazil. Int J Tuberc Lung Dis. 2013;17(10):S41-S47.

6. Figueiredo TMRM, Villa TCS, Scatena LM, Gonzales RIC, Ruffino-Netto A, Nogueira JA, et al. Desempenho da atenção básica no controle da tuberculose. RevSaúde Pública. 2009;43(5):825-31.

7. Marcolino ABL, Nogueira JÁ, Ruffino-Netto A, Moraes RM, Sá LD, Villa TCS, et al. Avaliação do acesso às ações de controle da tuberculose no contexto das equipes de saúde da família de Bayeux - PB. Rev. bras.

epidemiol. 2009;12(2):144-57.

8. Marquieviz J, Alves IS, Neves EB, Ulbricht L. A estratégia de saúde da família no controle da tuberculose em Curitiba (PR). Ciência\&SaúdeColetiva. 2013;18(1):265-71.

9. Organización Mundial de la Salud. Atención primaria de salud: Ahora más que nunca, OMS, Ginebra. 2008.

10. Instituto Brasileiro de Geografia e Estatística (IBGE). População estimada da cidade de Pelotas para o ano de 2014.

11. Ministério da Saúde. Departamento de atenção básica. Cobertura da estratégia de saúde da família, município de Pelotas/RS. 2014.

12. Villa TCS, Ruffino-Netto A. Questionário para avaliação de desempenho de serviços de atenção básica no controle da TB no Brasil. J Bras Pneumol. 2009;35(6):610-2.

13. Carvalho $\mathrm{H}$. Análise multivariada de dados qualitativos. Lisboa: Edições Silabo; 2004.

14. Le Roux B, Rouanet H. Geometric Data Analysis From Correspondence Analysis to Structured Data Analysis. Dordrecht, Kluwer Academic Publishers, 2004. 15. Guimarães RM, Lobo AP, Siqueira EA, Borges TFF, Melo SCC. TB, HIV e pobreza: tendência temporal no Brasil, Américas e mundo. J Bras Pneumol. 2012; 38(4):511-517.

16. Villa TCS, Ponce MAS, Wysocki AD, Andrade RLP, Arakawa T, Scatolin BE, Brunello MEF, Beraldo AA, Scatena LM, Monroe AA, Monroe AA, Sobrinho RAS; Sá LD, Nogueira JA, Assis MMA, Cardozo-Gonzales RI, Palha PF. Early diagnosis of tuberculosis in the health services in different regions of Brazil. Rev. Lat Am Enfermagem. 2013; 21:190-198.

17. Beduhn DAV, Harter J, Pieren SR, Barbosa AL, Cardozo-Gonzales RI. Desempeño laboratorial de las 
unidades de atención primaria en el diagnóstico de tuberculosis en Pelotas, Brasil. Rev Peru Med Exp Salud Publica. 2013;30(4):621-625.

18. Mendes JDV, Bittar OJNV. Perspectivas e Desafios da Gestão Pública no SUS. Revista da Faculdade de Ciências Médicas de Sorocaba. 2014;16(1):35-39.

19. Bastos LG, Fonseca LS, FC Mello, Ruffino-Netto A, Golub JE, Conde MB. Prevalência de TB pulmonar entre os indivíduos sintomáticos respiratórios em uma unidade básica de saúde ambulatorial. Int. J. Tuberc Lung Dis. 2007;11(2):156-60. Errata em: Int J Tuberc Lung Dis. 2007;11(8):936. Golub, JL [corrigido para Golub, JE] 20. Trigueiro DRSG, Nogueira JA, Monroe AA, Sá LD, Anjos UU, Villa TCS. Determinantes individuais e utilização dos serviços de saúde para o diagnóstico da TB. Rev. Enferm. UERJ. 2013;21(3):371-8.

21. Cardozo-Gonzales R, Costa L, Pereira C, Pinho L, Lima $L$, Soares $D$, et al. Ações de busca de sintomáticos respiratórios de TB na visão dos profissionais de uma unidade saúde da família. J Nurs Health. 2011;1(2):291-9.

Recebido: 18/11/2014.

Aceito: 20/08/2015.

Publicado: 31/12/2015. 